\title{
Narrow Band-Ultraviolet B Versus Clobetasol Propionate Foam in the Treatment of Vitiligo: A Retrospective Study
}

\author{
Giuseppe Stinco • Giusto Trevisan • Cinzia Buligan • \\ Giorgia Gregoraci · Sergio De Marchi • \\ Nicola di $\mathrm{Meo} \cdot$ Pasquale Patrone
}

To view enhanced content go to www.dermtherapy-open.com

Received: April 16, 2013 / Published online: June 4, 2013

(C) The Author(s) 2013. This article is published with open access at Springerlink.com

\section{ABSTRACT}

Introduction: Several therapeutic options are available for the treatment of vitiligo; among these phototherapy and topical steroids are the most widely documented. A topical formulation of $0.05 \%$ clobetasol propionate foam (CPF) has been introduced in the market, but no data are

G. Stinco $(\varangle) \cdot$ C. Buligan · P. Patrone

Department of Experimental and Clinical Medicine, Institute of Dermatology, University of Udine,

Udine, Italy

e-mail: giuseppe.stinco@uniud.it

G. Trevisan · S. De Marchi · N. di Meo Institute of Dermatology and Venereology,

University of Trieste, Trieste, Italy

\section{G. Gregoraci}

Section of Statistics, Department of Medical and Biological Sciences, University of Udine, Udine, Italy

\section{G. Gregoraci}

Department of Medical and Biological Sciences, Institute of Hygiene and Clinical Epidemiology, University of Udine, Udine, Italy

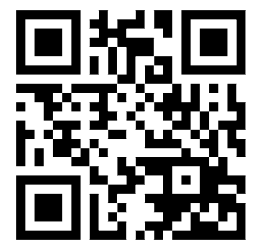

Enhanced content for this article is available on the journal web site: www.dermtherapy-open.com available about the efficacy and tolerability of this new formulation in the treatment of vitiligo. The aim of this study was to investigate the efficacy and tolerability of $\mathrm{CPF}$ in the treatment of vitiligo, in comparison with narrowband-ultraviolet B (NB-UVB) phototherapy.

Methods: The medical records of the first 60 vitiligo patients treated with NB-UVB phototherapy or with CPF were selected. Response to the treatment was determined for each anatomic site (neck, upper and lower limbs, trunk, hands/wrists, feet/ankles). Based on the area of repigmentation, treatment outcome was calculated according to a scale ranging from 0 (absent) to 4 (excellent). The incidence of adverse effects was also noted as a secondary endpoint. Significance level was set at $P=0.05$.

Results: For each anatomic site, statistical analyses demonstrated that the efficacy of CPF was significantly higher compared to NB-UVB. Side effects occurred in 4 patients $(13.33 \%)$ in the CPF group compared to none in the NBUVB group.

Discussion: Clobetasol propionate has been used in vitiligo in different vehicles, but never 
in foam. The data showed that CPF is effective and seems to be superior to NB-UVB phototherapy, with furthermore a good safety profile.

Conclusion: This new foam formulation of clobetasol propionate may expand the options currently available for vitiligo therapy; however, further investigations are needed to confirm our preliminary observations.

Keywords: Clobetasol propionate foam; Narrowband-ultraviolet B phototherapy; VersaFoam; Vitiligo

\section{INTRODUCTION}

The treatment of vitiligo is not well codified and often results are stressful and unsatisfying. A wide range of therapeutic options have been proposed including topical corticosteroids, ultraviolet radiations, either psoralens with ultraviolet A (PUVA) or narrowband-ultraviolet B (NB-UVB), lasers, surgical treatments, and more recently topical calcineurin inhibitors [1-3]. However, no treatment provides truly satisfactory results.

NB-UVB phototherapy is considered a firstline treatment for extensive vitiligo because of the relatively good efficacy and the excellent tolerance [4]. On the other hand, the topical steroids are indicated for the therapy of limited areas of vitiligo [5]. Clobetasol propionate is the most powerful of these drugs and it is available in the form of lotion, ointment, cream, emollient cream, solution, shampoo, and foam. A topical formulation of $0.05 \%$ clobetasol propionate foam (CPF; VersaFoam ${ }^{\circledR}$, Connetics, Palo Alto, CA, USA) has been available since 2006. Compared to cream and ointment this formulation leaves only a very little residue deposited on the skin with a better acceptability profile and with a greater positive effect on quality of life [6]. The cutaneous and systemic adverse effects of topical steroids depend on several factors including the activity, the systemic absorption and the bioavailability of the drug, the dose and the duration of the treatment. Clobetasol propionate cream or ointment has already been reported in the therapy of vitiligo [7-9], but no data are available regarding the use of foam formulation.

The aim of this study was to investigate the efficacy and tolerability of CPF in the treatment of vitiligo, in comparison with NB-UVB phototherapy.

\section{METHODS}

\section{Study Design}

The study subjects were selected from a group of adult patients with vitiligo vulgaris who were referred consecutively to the Departments of Dermatology of the Universities of Udine and Trieste in Italy from October 2009 to April 2010. For the purpose of this study, only patients who had completed the treatment protocol with CPF or NB-UVB were considered. To be included, the patients had to have performed a clinical evaluation before the start, at the half and at the end of therapy. Patients who had discontinued the treatment due to side effects were still recruited. Demographic data (age, sex, Fitzpatrick's skin phototype, comorbidities) and characteristics of vitiligo (pattern, location, duration, family history) had to be obtained from each patient. For each visit, data of physical examination and of possible adverse effects had to be obtained and digital photographs of the lesions taken both with normal ambient light and with Wood's lamp in standard pose. Patients who had received other treatments 
for vitiligo in the month prior to the start of CPF or NB-UVB were excluded from the study as well as those with infections, neurological or psychiatric disorders, immune defects, heart disease, renal failure, malignancy, history of photosensitivity or photomediated disorders, and a positive antinuclear antibody titer. Women who were pregnant and breastfeeding and patients taking known photosensitizer drugs or immunosuppressive therapy were also excluded from the study. The first 30 adult patients treated with UVB phototherapy and the first 30 treated with CPF who fulfilled these criteria were included in the study. All patients had nonsegmental vitiligo for almost 1 year (chronic) without any new depigmented patches in the past 12 months (stable).

All procedures followed were in accordance with the ethical standards of the responsible committee on human experimentation (institutional and national) and with the Helsinki Declaration of 1975 , as revised in 2000 and 2008. All the patients had given informed written consent to the treatment and to the photos.

According to the treatment received, patients were categorized into two groups (A and $\mathrm{B}$ ); patients in group A had undergone NBUVB phototherapy while group B patients had received CPF [VersaFoam-hydroethanolic foam (HF) formulation containing 60\% ethanol].

Twenty-two patients (36.6\%), 6 in group A and 16 in group B, had never been treated for vitiligo, while the remaining 38 patients had previously received treatment with other topical steroids, calcineurin inhibitors, PUVA, and topical calcipotriol with unsatisfactory results.

\section{Therapeutic Regimen}

Group A patients had been treated with NBUVB as monotherapy using the N-LINE pro cabin (Saalmann medical GmbH, Hiddenhausen, Germany) with a digital timer. Phototherapy had been given thrice a week on non-consecutive days, for a maximum of 6 months or 50 sessions in total. Initial phototesting had not been done. The minimal erythematous dose (MED) was pre-determined $\left(280 \mathrm{~mJ} / \mathrm{cm}^{2}\right)$ according to the concept that the depigmented skin lesions of vitiligo are considered as phototype I [10]. The initial dose in all patients was $280 \mathrm{~mJ} / \mathrm{cm}^{2}$, with subsequent $15 \%$ increments from the previous dosage in every session until minimal erythema appeared. Thereafter, the dose had been maintained until the sixth month therapy completion or till a complete repigmentation was achieved, whichever occurred first. In case of symptomatic erythema (burning, pain) or blistering, the irradiation dose had been decreased by $15 \%$. Standard photo-protection protocol for NB-UVB had been observed. During treatment, the genital area had been shielded and the eyes are protected by UV-blocking goggles. Barring these protected areas, wholebody irradiation was performed. In case of vitiligo present in the eyelid area, patients were asked not to wear goggles but had been advised to keep their eyes shut during the sessions. Patients were advised to apply a very high protection sunscreen with frequent reapplication and incorporation of sun avoidance techniques (avoidance of midday sun and wearing a hat).

Group B patients had been instructed to apply CPF, with a fine massage until the product was fully absorbed, the smallest amount of foam necessary to cover all the lesions twice a day for 5 days/week for 12 weeks. Subjects dispensed foam into the cap of the container and applied a small amount of foam to the vitiligo lesions. One cap of foam weighs $3.5 \mathrm{~g}$, the maximum amount the subject could use for each 
application. When one cup of foam was not sufficient to cover all the lesions, for safety reasons, the patients were suggested to treat only the anatomical site they considered the most disturbing from an esthetic and psychological point of view. Face and intertriginous areas were excluded from the treatment and patients were also asked to stop the treatment in case of occurrence of side effects such as infection signs, atrophy, teleangectasy, and folliculitis. All patients were also asked to avoid the direct UV exposition during the whole period of study and were advised to apply a very high protection sunscreen with frequent reapplication and incorporation of sun avoidance techniques (avoidance of midday sun and wearing a hat).

\section{Clinical Evaluation}

The digital lesional photographs, both with normal ambient light and with Wood's lamp obtained in a standard pose before the start, at the half and at the end of therapy were examined visually by two different dermatologists and evaluated through singlecase discussion between these two experts. Response to the treatment was determined for each anatomic site (neck, upper and lower limbs, trunk, hands/wrists, feet/ankles) by assigning the entire lesion a $0 \%$ score before treatment to indicate a baseline of no repigmentation and a second percentage value at the end of the study to represent the level of repigmentation. The area of repigmentation was analyzed by serial mapping of body lesions. Treatment outcome was calculated for each anatomic site according to a scale ranging from 0 to 4 and classified as ' 0 , absent' ( $0 \%)$, ' 1 , poor' (1-25\%), '2, moderate' (26-50\%), '3, good' (51-75\%), and '4, excellent' (>75\%) performing a visual comparison with a pre-treatment photography under the same lighting conditions.

Data about potential repigmentation due to NB-UVB of face and intertriginous areas were not reported because in the CPF group those anatomic sites have not been treated since the risk of severe side effects linked to the twicedaily application of a superpotent steroid. During the whole period of the study, any possible side effects were recorded.

\section{Statistical Analysis}

Characteristics of the study population are described using mean and standard deviation or median and range for continuous or ordinal variables and as frequencies and percentages for categorical variables. Normality was assessed with the Shapiro-Wilk test. Analyses for the efficacy of each treatment were based on the comparison between the before treatment assessment and the last clinical evaluation. Comparisons between treatment groups were performed using $T$ test or Mann-Whitney rank test for continuous or ordinal variables based on the results of the Shapiro-Wilk test, and Chisquare or Fisher's exact test for categorical variables, as appropriate. Significance level was set at $P=0.05$. All analyses were performed using Stata ${ }^{\circledR}$ (version SE 12.1, Statacorp LP, College Station, TX, USA).

\section{RESULTS}

\section{Patient Characteristics}

Baseline characteristics of the study population are summarized in Table 1 . The mean age of the patients treated with CPF was slightly lower compared with those who had received NBUVB. There was no significant difference in the 
distribution of sex, pattern, Fitzpatrick's skin phototypes, and family history of vitiligo between the NB-UVB treated group and the CPF-treated group. The duration of vitiligo was significantly shorter in the NB-UVB group compared with the CPF group. Among the comorbidities, thyroid diseases were more frequent in the CPF-treated group.

\section{Comparison Between NB-UVB and CPF Treatments}

The results of NB-UVB and CPF treatments in patients with vitiligo are summarized in Table 2. The number of patients treated for each anatomic site was homogeneous between the two groups.

Overall, for each site, the efficacy of CPF was significantly higher compared with NB-UVB (Fig. 1). In the CPF-treated group, a variable repigmentation was found, from poor to excellent, at neck, upper and lower limbs, trunk, and hand/wrists (Fig. 2). Lack of repigmentation was observed at feet/ankles in 6 of 11 treated patients. In the NB-UVB group at feet/ankles, no sign of repigmentation was found in any of the 12 treated patients. In addition, lack of repigmentation was observed at hands/wrists in 13 of 16 treated patients. In the remaining anatomical sites, variable levels of repigmentation were recorded. In the NBUVB group, none of the patients reported adverse effects throughout the study period. In the CPF group, a woman stopped treatment due to the appearance of diffuse edema. Three other patients reported a slight and transient erythema with itching which disappeared after the next application without having to discontinue the treatment. The difference in occurrence of side effects observed in the two groups was not significant (Fisher's test $P=0.112$ )

\section{DISCUSSION}

Several therapeutic options have been proposed for vitiligo, but successful repigmentation occurs only in about half of the treated patients. Topical and systemic corticosteroids, topical calcineurin inhibitors, calcipotriol, phototherapy, excimer laser and surgical methods, such as skin/single-hair grafting, or autologous cultured melanocyte or epidermal suspension transplantations have been used with different results [2, 3]. Among these treatments, phototherapy and topical steroids are the most widely documented $[5,10]$.

Several studies have been published on the NB-UVB treatment of vitiligo in different populations of patients with variable, but usually good, responses [11-16]. Overall NBUVB was accepted and well tolerated by most patients. The most common short-time side effects include erythema, pruritus, and xerosis; moreover, NB-UVB has been known to cause phototoxic reactions and tanning. There are little long-term data on the use of UVB in the treatment of vitiligo, but one potential adverse effect could be an increased chance of nonmelanoma skin cancer in vitiliginous skin receiving NB-UVB [4]. The clinical efficacy of clobetasol propionate has been known for many years for the treatment of vitiligo [17, 18]. A topical formulation of this superpotent corticosteroid in thermophobic foam has been reported as an effective treatment in psoriasis [19], chronic hand dermatitis [20], atopic dermatitis [21], alopecia areata [22], and delayed pressure urticaria [23]. Clobetasol propionate has been used in vitiligo in different vehicles, but never in foam. The results of this study demonstrate that in a population of patients with vitiligo CPF can be an effective option and its clinical efficacy seems to be superior to NB-UVB phototherapy. 
Till now, to the best of the authors' knowledge, no study of comparison between UVB and clobetasol propionate has been published. Buggiani et al. [24] compared subjects treated with UVB microphototherapy and with clobetasol propionate ointment. Excellent repigmentation (>75\%) was achieved by $61.1 \%$ of patients in the UVB microphototherapy group and by $56.2 \%$ of patients in the clobetasol propionate ointment group. In the CPF-treated group, the median repigmentation scores on neck, upper limbs, lower limbs, and trunk were significantly higher than in the NBUVB group in the same body sites. For the hand/

Table 1 Characteristics of the study population

\begin{tabular}{|c|c|c|c|}
\hline & $\begin{array}{l}\text { Patients treated } \\
\text { with NB-UVB } N(\%)\end{array}$ & $\begin{array}{l}\text { Patients treated } \\
\text { with CPF } N(\%)\end{array}$ & $P$ value \\
\hline Mean age $\pm S D$ (years) & $47.2 \pm 14.6$ & $39.8 \pm 13.6$ & $0.049^{\mathrm{a}}$ \\
\hline Male/female & $16 / 14$ & $12 / 18$ & $0.301^{\mathrm{c}}$ \\
\hline \multicolumn{4}{|l|}{ Pattern of vitiligo } \\
\hline Only nonsegmental & $30(100)$ & $30(100)$ & \\
\hline Acrofacial & $1(3.3)$ & $1(3.3)$ & $1.000^{\mathrm{d}}$ \\
\hline Generalized & $17(56.7)$ & $16(53.3)$ & \\
\hline Localized & $12(40.0)$ & $13(43.3)$ & \\
\hline Median duration of disease, years(interquartile range) & $4.0(3-23)$ & $8.5(4-20)$ & $0.028^{\mathrm{b}}$ \\
\hline \multicolumn{4}{|l|}{ Fitzpatrick's skin phototype } \\
\hline Phototype I & $0(0)$ & $0(0)$ & \\
\hline Phototype II & $4(13.3)$ & $11(36.7)$ & \\
\hline Phototype III & $14(46.7)$ & $13(43.3)$ & $0.125^{\mathrm{d}}$ \\
\hline Phototype IV & $8(26.7)$ & $3(10)$ & \\
\hline Phototype V & $4(13.3)$ & $3(10)$ & \\
\hline \multicolumn{4}{|l|}{ Family history of vitiligo } \\
\hline Yes & $8(26.7)$ & $5(16.7)$ & $0.532^{c}$ \\
\hline No & $22(73.3)$ & $25(83.3)$ & \\
\hline \multicolumn{4}{|l|}{ Comorbidities } \\
\hline None & $24(80)$ & $19(63.3)$ & \\
\hline Thyroid disease & $1(3.3)$ & $9(30)$ & $0.020^{\mathrm{d}}$ \\
\hline Others & $5(16.7)$ & $2(6.7)$ & \\
\hline
\end{tabular}

$N B-U V B$ narrow band-ultraviolet B phototherapy, $C P F 0.05 \%$ clobetasol propionate foam

${ }^{a} T$ test

${ }^{b}$ Mann-Whitney test

${ }^{c}$ Chi-square test

${ }^{\mathrm{d}}$ Fisher's test 
Table 2 Clinical efficacy of NB-UVB and CPF treatments for each body site

\begin{tabular}{|c|c|c|c|}
\hline & $\begin{array}{l}\text { Patients treated } \\
\text { with NB-UVB } N(\%)\end{array}$ & $\begin{array}{l}\text { Patients treated } \\
\text { with CPF } N(\%)\end{array}$ & $P$ value \\
\hline \multicolumn{4}{|l|}{ Neck } \\
\hline Number of treated patients (\%) & $10(33.3)$ & $8(26.7)$ & $0.573^{\mathrm{b}}$ \\
\hline Repigmentation score, median (interquartile range) & $2(1-3)$ & $4(2-4)$ & $0.005^{\mathrm{a}}$ \\
\hline \multicolumn{4}{|l|}{ Upper limbs } \\
\hline Number of treated patients (\%) & $17(56.7)$ & $16(53.3)$ & $0.795^{\mathrm{b}}$ \\
\hline Repigmentation score, median (interquartile range) & $1(1-3)$ & $3(1-4)$ & $0.002^{\mathrm{a}}$ \\
\hline \multicolumn{4}{|l|}{ Hand/wrists } \\
\hline Number of treated patients (\%) & $15(50)$ & $19(63.3)$ & $0.297^{\mathrm{b}}$ \\
\hline Repigmentation score, median (interquartile range) & $0(0-2)$ & $1(1-2)$ & $<0.0001^{\mathrm{a}}$ \\
\hline \multicolumn{4}{|l|}{ Trunk } \\
\hline Number of treated patients (\%) & $12(40)$ & $12(40)$ & $1.000^{\mathrm{b}}$ \\
\hline Repigmentation score, median (interquartile range) & $2(1-2)$ & $3(1-4)$ & $0.008^{\mathrm{a}}$ \\
\hline \multicolumn{4}{|l|}{ Lower limbs } \\
\hline Number of treated patients (\%) & $15(50)$ & $11(36.7)$ & $0.297^{\mathrm{b}}$ \\
\hline Repigmentation score, median (interquartile range) & $1(1-2)$ & $3(2-3)$ & $<0.0001^{\mathrm{a}}$ \\
\hline \multicolumn{4}{|l|}{ Feet/ankles } \\
\hline Number of treated patients (\%) & $12(40)$ & $11(36.7)$ & $0.791^{\mathrm{b}}$ \\
\hline Repigmentation score, median (interquartile score) & $0(0)$ & $0(0-1)$ & $0.010^{\mathrm{a}}$ \\
\hline
\end{tabular}

NB-UVB narrowband-ultraviolet B phototherapy, CPF $0.05 \%$ clobetasol propionate foam

${ }^{a}$ Mann-Whitney test

${ }^{b}$ Chi-square test

wrists and feet/ankles, despite that both groups showed low repigmentation levels, the median scores were significantly higher in patients treated with CPF. The location of lesions is the most important factor in predicting the response of vitiligo to therapy and previous studies had already shown that face and neck achieve better repigmentation than trunk and extremities [16, 25, 26]. Another factor that may contribute to a good response is the shorter duration of disease [25]. Interestingly, in this study the topical steroid therapy showed a better clinical response, although the duration of disease in the CPF group was significantly longer as compared to the NB-UVB group. This finding seems to confirm the good efficacy of the new topical formulation of this superpotent corticosteroid in patients with vitiligo.

The chronic use of topical corticosteroids might cause side effect as skin atrophy, striae, hypertrichosis, acneiform eruption, and telangiectasia which are limiting factors in vitiligo treatment [27]. CPF has been proposed for long-term therapy in chronic dermatosis [19-23]. Lacarrubba et al. [28] evaluated through ultrasound imaging the atrophogenic 


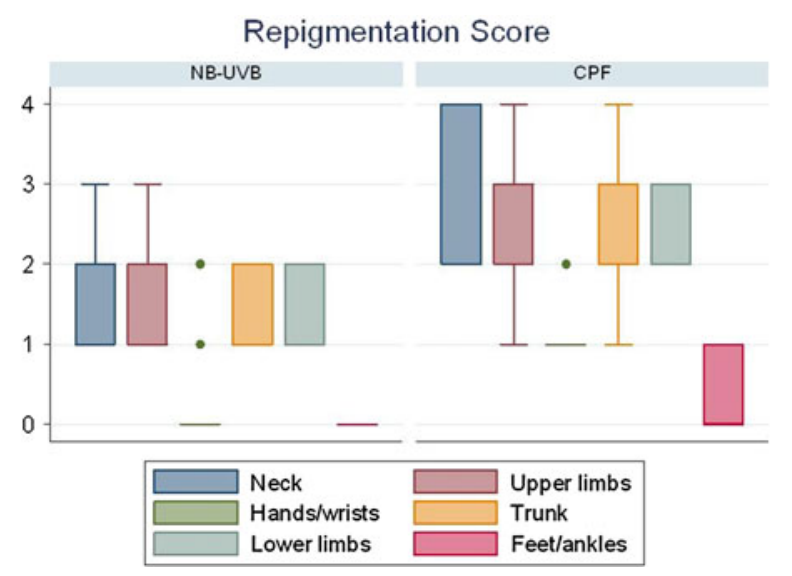

Fig. 1 Median repigmentation scores for anatomic sites, by treatment regimen. Treatment outcome scores were ' 0 , absent' (0\%), '1, poor' (1-25\%), '2, moderate' (26-50\%), ' 3 , good' (51-75\%), and ' 4 , excellent' (>75\%)

potential of once-daily application of CPF on healthy skin and they did not observe any ultrasound variations in skin thickness. Recent studies treated vitiligo patients with $0.05 \%$ clobetasol propionate ointment, twice a day for 12 weeks with mild to moderate side effects [24]. In the present study, the patients applied CPF twice a day for 5 days/week for 12 weeks. This therapeutic protocol has already been proposed in alopecia areata by Tosti et al. [22] who demonstrated the safety of this scheme. Overall in this study the adverse effects were rare. No adverse effect was reported in the NBUVB group. In the CPF-treated group, three patients reported a slight and transient erythema with itching which disappeared after the next application and only in one woman the treatment had to be stopped due to the appearance of diffuse edema. Anyway the difference in occurrence of side effects observed in the two groups of treatment was not significant. Therefore, these findings suggest that in adult patients with vitiligo, CPF and NB-UVB phototherapy are effective treatment options with a good safety profile. $\mathrm{CPF}$ has some additional benefits compared with NB-UVB phototherapy; the treatment can be self-managed by the patient and the results of a good/excellent repigmentation require less time to manifest (up to 3 months with $\mathrm{CPF}$ versus up to 6 months with NB-UVB). All these factors should be kept in mind when considering the patient's adherence to treatment which is likely the most important determinant of the therapeutic success.

This study is the first, to the best of the authors' knowledge, that evaluates the clinical efficacy of the new foam vehicle of $0.05 \%$ clobetasol propionate in the treatment of vitiligo. Its efficacy and safety, the ease of application, and relatively low cost of the therapy prompted us to introduce this CPF formulation in the treatment of vitiligo.
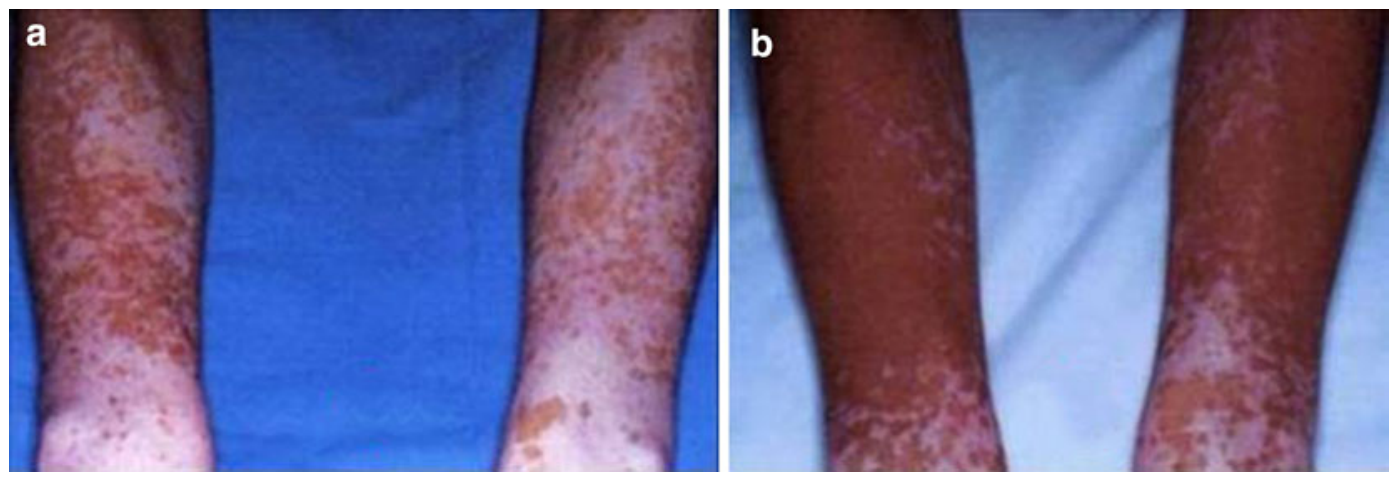

Fig. 2 Vitiligo of the forearms before (a) and after (b) treatment with $0.05 \%$ clobetasol propionate foam 


\section{CONCLUSION}

The findings suggest a good efficacy of the CPF in vitiligo in all the treated anatomic sites compared to the NB-UVB phototherapy and a good safety profile in a medium-term use. This new foam formulation may expand the options currently available for vitiligo therapy; however, further investigations are needed to confirm the preliminary observations. Since clobetasol propionate is a well-known treatment for vitiligo, and the results seems to demonstrate the efficacy of the novel formulation of this drug, in the future, it would be reasonable to compare traditional clobetasol propionate formulations against CPF to deepen the advantages brought by the foam vehicle. It would also be interesting to know the potential advantage introduced by the new formulation of the foam (VersaFoam-EF emollient foam technology which does not contain any ethanol). Furthermore, combined treatments seem to be presently superior to monotherapies in terms of efficacy and safety. It is an established fact that combination of NBUVB with topical treatment(s) is superior over monotherapy [29]; therefore, further studies would be opportune to evaluate not only the relative efficiency of and patient's compliance to CPF against traditional clobetasol propionate formulations alone, but also when combined with NB-UVB.

\section{BULLET POINTS}

- Recently a new topic formulation of $0.05 \%$ clobetasol propionate in thermophobic foam (CPF) has been reported as an effective treatment in several dermatoses, but no data are reported in vitiligo.

- Based on the results of a retrospective study on 60 vitiligo patients, we discuss the efficacy and tolerability of CPF compared to NB-UVB phototherapy.

- $\mathrm{CPF}$ is effective and seems to be superior to NB-UVB phototherapy, with a good safety profile.

- This is the first study that investigates the clinical efficacy of CPF in the treatment of vitiligo.

\section{ACKNOWLEDGMENTS}

No funding or sponsorship was received for this study or publication of this article. This was an industry-independent study and no conflict of interest concerning sponsorship of any kind was noted. Dr. G. Stinco is the guarantor for this article and takes responsibility for the integrity of the work as a whole.

Conflict of interest. Drs. G. Stinco, G. Trevisan, C. Buligan, G. Gregoraci, S. De Marchi, N. di Meo, P. Patrone declare they have no conflict of interest.

Compliance with ethics guidelines. All procedures followed were in accordance with the ethical standards of the responsible committee on human experimentation (institutional and national) and with the Helsinki Declaration of 1975 , as revised in 2000 and 2008. Informed consent was obtained from all patients for their treatment and photos.

Open Access. This article is distributed under the terms of the Creative Commons Attribution Noncommercial License which permits any noncommercial use, distribution, and reproduction in any medium, provided the original author(s) and the source are credited. 


\section{REFERENCES}

1. Colucci R, Lotti T, Moretti S. Vitiligo: an update on current pharmacotherapy and future directions. Expert Opin Pharmacother. 2012;13:1885-99.

2. Bacigalupi RM, Postolova A, Davis RS. Evidencebased, non-surgical treatments for vitiligo: a review. Am J Clin Dermatol. 2012;13:217-37.

3. Hossani-Madani A, Halder R. Treatment of vitiligo: advantages and disadvantages, indications for use and outcomes. G Ital Dermatol Venereol. 2011;146:373-95.

4. Hamzavi IH, Lim HW, Syed ZU. Ultraviolet-based therapy for vitiligo: what's new? Indian J Dermatol Venereol Leprol. 2012;78:42-8.

5. Forschener T, Bucholtz S, Stockfleth E. Current state of vitiligo therapy-evidence-based analysis of the literature. J Dtsch Dermatol Ges. 2007;5:467-75.

6. Stein L. Clinical studies of a new vehicle formulation for topical corticosteroids in the treatment of psoriasis. J Am Acad Dermatol. 2005;53:39-49.

7. Handa S, Pandhi R, Kaur I. Vitiligo: a retrospective comparative analysis of treatment modalities in 500 patients. J Dermatol. 2001;28:461-6.

8. Coskun B, Saral Y, Turgut D. Topical $0.05 \%$ clobetasol propionate versus $1 \%$ pimecrolimus ointment in vitiligo. Eur J Dermatol. 2005;15:88-91.

9. Ho N, Pope E, Weinstein M, Greenberg S, Webster C, Krafchik BR. A double-blind, randomized, placebo-controlled trial of topical tacrolimus $0.1 \%$ vs. clobetasol propionate $0.05 \%$ in childhood vitiligo. Br J Dermatol. 2011;165:626-32.

10. Scherschun L, Kim JJ, Lim HW. Narrow-band ultraviolet $\mathrm{B}$ is a useful and well-tolerated treatment for vitiligo. J Am Acad Dermatol. 2001;44:999-1003.

11. Dong D, Jiang M, Xu X, et al. The effects of NB-UVB on the hair follicle-derived neural crest stem cells differentiating into melanocyte lineage in vitro. J Dermatol Sci. 2012;66:20-8.

12. De Francesco V, Stinco G, Laspina S, Parlangeli ME, Mariuzzi L, Patrone P. Immunohistochemical study before and after narrow band $(311 \mathrm{~nm})$ UVB treatment in vitiligo. Eur J Dermatol. 2008;18:292-6.

13. Korobko IV. Review of current clinical studies of vitiligo treatments. Dermatol Ther. 2012;25 Suppl:17-27.
14. Hamzavi I, Jain H, McLean D, Shapiro J, Zeng H, Lui $\mathrm{H}$. Parametric modeling of narrowband UV-B phototherapy for vitiligo using a novel quantitative tool: the Vitiligo Area Scoring Index. Arch Dermatol. 2004;140:677-83.

15. Sapam R, Agrawal S, Dhali TK. Systemic PUVA vs. narrowband UVB in the treatment of vitiligo: a randomized controlled study. Int J Dermatol. 2012;51:1107-15.

16. Stinco G, Piccirillo F, Forcione M, Valent F, Patrone $P$. An open randomized study to compare narrow band UVB, topical pimecrolimus and topical tacrolimus in the treatment of vitiligo. Eur J Dermatol. 2009;19:588-93.

17. Clayton R. A double-blind trial of $0-05 \%$ clobetasol propionate in the treatment of vitiligo. $\mathrm{Br} \mathrm{J}$ Dermatol. 1977;96:71-3.

18. Kumari J. Vitiligo treated with topical clobetasol propionate. Arch Dermatol. 1984;120:631-5.

19. Mazzotta A, Esposito M, Carboni I, Schipani C, Chimenti S. Clobetasol propionate foam $0.05 \%$ as a novel topical formulation for plaque-type and scalp psoriasis. J Dermatol Treat. 2007;18:84-7.

20. Kircik LH, Tropmann C. Treatment of mild-tomoderate chronic hand dermatitis with clobetasol propionate $0.05 \%$ EF foam: results from an openlabel study. J Drugs Dermatol. 2011;10:1398-402.

21. Kimball AB, Gold MH, Zib B, Davis MW, Clobetasol Propionate Emulsion Formulation Foam Phase III Clinical Study Group. Clobetasol propionate emulsion formulation foam $0.05 \%$ : review of phase II open-label and phase III randomized controlled trials in steroid-responsive dermatoses in adults and adolescents. J Am Acad Dermatol. 2008;59:448-54.

22. Tosti A, Iorizzo M, Botta GL, Milani M. Efficacy and safety of a new clobetasol propionate $0.05 \%$ foam in alopecia areata: a randomized, double-blind placebo-controlled trial. J Eur Acad Dermatol Venereol. 2006;20:1243-7.

23. Vena GA, Cassano N, D'Argento V, Milani M. Clobetasol propionate $0.05 \%$ in a novel foam formulation is safe and effective in the short-term treatment of patients with delayed pressure urticaria: a randomized, double-blind, placebocontrolled trial. Br J Dermatol. 2006;154:353-6.

24. Buggiani $G$, Tsampau $D$, Hercogovà $J$, Rossi R, Brazzini B, Lotti T. Clinical efficacy of a novel topical formulation for vitiligo: compared evaluation of different treatment modalities in 149 patients. Dermatol Ther. 2012;25:472-6. 
25. Kanwar AJ, Dogra S, Parsad D, Kumar B. Narrowband UVB for the treatment of vitiligo: an emerging effective and well-tolerated therapy. Int J Dermatol. 2005;44:57-60.

26. Kishan Kumar YH, Rao GR, Gopal KV, Shanti G, Rao KV. Evaluation of narrow-band UVB phototherapy in 150 patients with vitiligo. Indian J Dermatol Venereol Leprol. 2009;75:162-6.

27. Borderè AC, Lambert $\mathrm{J}$, van Geel N. Current and emerging therapy for the management of vitiligo. Clin Cosmet Investig Dermatol. 2009;2:15-25.
28. Lacarrubba F, Nardone B, Musumeci ML, Micali G. Ultrasound evaluation of clobetasol propionate $0.05 \%$ foam application in psoriatic and healthy skin: a pilot study. Dermatol Ther. 2009;22:19-21.

29. Lotti T, Buggiani G, Troiano M, Assad GB, Delescluse J, De Giorgi V, Hercogova J. Targeted and combination treatments for vitiligo. Comparative evaluation of different current modalities in 458 subjects. Dermatol Ther. 2008;21:20-6. 